Cahiers $d u$ MONDE RUSSE

\section{Cahiers du monde russe}

Russie - Empire russe - Union soviétique et États indépendants

$58 / 4 \mid 2017$

Varia

\title{
Olga Medvedkova, éd., Pierre le Grand et ses livres : Les arts et les sciences de l'Europe dans la bibliothèque du Tsar
}

\section{Pierre Caye}

\section{(2) OpenEdition}

12 Journals

Édition électronique

URL : http://journals.openedition.org/monderusse/10147

DOI : $10.4000 /$ monderusse. 10147

ISSN : $1777-5388$

Éditeur

Éditions de l'EHESS

Édition imprimée

Date de publication : 1 octobre 2017

Pagination : 681-684

ISBN : 978-2-7132-2698-4

ISSN : $1252-6576$

Référence électronique

Pierre Caye, «Olga Medvedkova, éd., Pierre le Grand et ses livres : Les arts et les sciences de l'Europe dans la bibliothèque du Tsar », Cahiers du monde russe [En ligne], 58/4 | 2017, mis en ligne le 01 octobre 2017, consulté le 06 janvier 2021. URL : http://journals.openedition.org/monderusse/10147 DOI : https://doi.org/10.4000/monderusse.10147 
Olga MEDVEDKOVA, éd.,

\section{Pierre le Grand et ses livres}

\section{Les arts et les sciences de l'Europe dans la bibliothèque du Tsar,}

Paris : CNRS - Alain Baudry et Cie (Respublica Literaria), 2016, 750 p.

Le catalogue raisonné de la bibliothèque du tsar Pierre ${ }^{\text {er }}$ de Russie, plus connu sous le nom de Pierre le Grand, que publient les éditions Alain Baudry sous la direction d'Olga Medvedkova, directrice de recherche au CNRS, est un outil précieux pour comprendre non seulement le règne de Pierre le Grand, mais plus profondément encore les rapports entre savoir et pouvoir dans l'Europe moderne. La série d'études de Wladimir Berelowitch, Olga Medvedkova, Philippe Malgouyres, Ernest Zitser, Pascal Dubourg Glatigny, Emilie d'Orgeix, Irina Khmelevskikh, qui précèdent le catalogue proprement dit établi par Irina Khmelevskikh apporte à cet égard les plus grands éclaircissements. En effet, la bibliothèque de Pierre le Grand n'intéresse pas seulement le bibliophile, l'historien du livre ou l'historien de l'art, mais elle est d'abord un objet épistémologique et politique.

Elle est d'abord un objet épistémologique en ce que la bibliothèque est ici essentiellement au service de la constitution d'un savoir, qui plus est d'un savoir opératoire. Les savoirs à l'âge moderne ne sont évidemment pas aussi fortement constitués qu'à partir du $\mathrm{XIX}^{\mathrm{e}}$ siècle ; les frontières sont plus souples, les méthodes plus incertaines, leur approche moins disciplinaire. Ils revêtent, par conséquent, un caractère plus encyclopédique : pour comprendre le réel, il est nécessaire d'entrecroiser les connaissances. Et il faut, à partir de cet entrecroisement, inventer son savoir. La bibliothèque est le lieu de cette invention, à la fois carrefour et creuset de savoirs autour d'un projet cohérent, en l'occurrence l'aménagement du territoire, l'édification de Saint-Pétersbourg ainsi que plus généralement l'organisation administrative et militaire de la monarchie russe. Ce qui explique la nature même de cette bibliothèque qui n'a rien d'ostentatoire, qui n'est aucunement, au contraire des autres grandes bibliothèques princières, l'expression d'un trésor symbolique ou encore d'une accumulation culturelle patrimoniale, mais qui se veut d'abord un atelier, un laboratoire. Les livres de cette bibliothèque bénéficient très rarement de reliures de prestige, mais on y trouve beaucoup de livres annotés, découpés, démembrés, constamment manipulés et transportés, signe d'un savoir vivant et heuristique (v. Emilie d'Orgeix, Pierre le Grand, sa bibliothèque nomade et ses lectures martiales, p. 91). À l'exemple de Vauban qui lui-même avait aménagé dans son carrosse une petite bibliothèque portative l'accompagnant dans ses visites d'inspection de la ceinture de fer, Pierre le Grand faisait mettre une partie de ses livres en caisse pour l'accompagner dans ses propres déplacements.

La bibliothèque, en particulier celle de Pierre le Grand, n'est pas simplement une concentration de livres, mais est aussi une convergence de langues. Olga Medvedkova dans son étude (Les livres du Tsar, p. 32-33) note avec pertinence la singularité de la langue de Pierre le Grand, sa polyglossie, parfois macaronique, qui mêle latin, hollandais, russe pour essayer de dire ses pensées, comme si son mode de réflexion excédait ce que la langue russe lui permettait de formuler. De fait, cette 
bibliothèque organise une véritable circulation linguistique, un champ de traduction, non seulement de traduction vers le russe, pour permettre à celui-ci de devenir à son tour une langue scientifique et technique, mais aussi et surtout de traduction tous azimuts entre les diverses langues de l'Europe occidentale, puisque cette bibliothèque comporte beaucoup d'ouvrages d'auteurs français ou italiens mais, et la précision est importante, dans leur traduction allemande ou hollandaise. Les langues de l'Europe dans la bibliothèque de Pierre le Grand finissent par se contaminer les unes les autres. Ce rôle central de la traduction dont témoigne cette bibliothèque marque assurément l'importance de la langue dans la constitution du savoir à l'âge classique : le savoir classique est une sémantique : nous sommes encore, à ce moment, dans un état du savoir scientifique où les mots, les noms importent plus que les nombres.

Et quand les mots n'arrivent pas à dire le réel, alors Pierre le Grand a recours au dessin d'où le fait que cette bibliothèque est aussi un cabinet d'estampes et de dessins. Le dessin fait pont entre les mots et les choses. De sorte que la bibliothèque de Pierre le Grand, comme bien d'autres bibliothèques de son temps, met en valeur la fameuse triade galénique qui joue un rôle fondamental dans le savoir humaniste et classique, à savoir la triade logos/skia/ergon, autrement dit réflexion (logos), dessin (skia), opération ou œuvre (ergon), triade qui est en réalité bijective, c'est-à-dire qui circule dans les deux sens : le dessin sert autant à transmettre les leçons de la pratique à la pensée théorique qu'à traduire, dans la réalité physique, les conceptions de la pensée. Et c'est précisément parce qu'elle circule dans les deux sens que cette triade est si féconde.

La bibliothèque de Pierre le Grand est aussi un objet politique. Le savoir classique est une sémantique, avons-nous dit. Il est une seconde thèse que la bibliothèque de Pierre le Grand semble nous autoriser à poser : l'État moderne repose sur le livre, et c'est en quoi la formation de cet État appartient encore à l'histoire de l'humanisme. C'est vrai de la Russie de Pierre le Grand, comme de la monarchie des Bourbons, mais cela le reste, un siècle après Pierre le Grand, aux États-Unis, quelques années à peine après la déclaration d'indépendance, si l'on prend la mesure de la bibliothèque du président Thomas Jefferson, qui sert de point de départ à la bibliothèque du Congrès et qui ressemble, par bien des traits, à celle de Pierre le Grand, dans un cadre où, là aussi, l'architecture joue le plus grand rôle. On sait qu'en même temps que Jefferson constituait sa bibliothèque, il faisait construire l'université de Charlottesville ; on sait combien d'une façon générale les bibliothèques occupent une place importante dans les grandes universités américaines; on sait enfin combien ces grandes universités ont joué et continuent à jouer un rôle fondamental dans la construction et surtout dans l'unification de la nation américaine, à travers en particulier sa formation des élites. Ce parallélisme entre les deux grandes puissances nouvelles de l'Occident, la Russie et les États-Unis, leur rivalité dans l'expression de la modernisation de l'Europe, se poursuivra jusqu' au $\mathrm{XX}^{\mathrm{e}}$ siècle. Assurément, la bibliothèque de Pierre le Grand est au service de la construction de la monarchie militaro-administrative russe, ou mieux encore au service de la raison d'État, non pas certes au sens d'Alexandre 
Dumas et de la légende noire du cardinal de Richelieu, mais au sens propre que lui a donné le père jésuite Giovanni Botero dans son ouvrage de 1589 justement intitulé la raison d'État, autrement dit les savoirs et les techniques utilisés par l'administration en vue de rendre la nation plus puissante. Le livre de Botero pourrait s'intituler La puissance des nations de même que, deux siècles plus tard, Adam Smith écrira La Richesse des nations. Car Pierre le Grand témoigne encore d'une époque où la puissance est cause de la richesse plus que la richesse cause de la puissance.

L'ouvrage dirigé par Olga Medvedkova nous permet d'avoir une connaissance précise du contenu de cette bibliothèque et par là même une compréhension plus approfondie de l'objet à la fois épistémologique et politique qu'elle constitue. Son contenu en effet est particulièrement cohérent et significatif; il marque l'étroite convergence, propre à l'âge humaniste et classique, des arts, des sciences et des techniques: s'y mêlent donc des ouvrages de mathématiques et de jardinage, de fortification et d'ornement, d'hydraulique et d'architecture civile, etc. À cet égard, la bibliothèque de Pierre le Grand présente une triple caractéristique particulièrement éclairante pour la signification de cette convergence et pour l'évolution des savoirs opératoires à l'âge classique :

Olga Medvedkova (cit. p. 36) puis Philippe Malgouyres (Les livres français $d u$ Tsar, p. 62) notent à juste titre l'importance considérable de l'architecture navale qui leur apparaît comme un véritable paradigme de la technique pour le modèle russe de développement que Pierre le Grand essaie de mettre sur pied. Olga Medvedkova insiste aussi sur la place de l'Amirauté qui concentre les savoir-faire les plus pointus et les ouvriers les plus compétents. Il existe entre l'Amirauté et la bibliothèque de Pierre le Grand le même rapport que Vitruve trace entre la Fabrica et la Ratiocinatio, le chantier et le projet. L'Amirauté fournit les instruments de la réalisation, et la bibliothèque ceux de la conception. Mais que signifie que l'architecture navale est le paradigme de la technique ? Quel est le sens de ce passage de relais de l'architecture vitruvienne à l'architecture navale comme paradigme de la technique? L'architecture vitruvienne fait arche : la technique ici met l'homme à distance de la nature, elle l'en protège. L'architecture navale, quant à elle, entretient un rapport plus étroit et plus synergique avec la nature, elle produit des véhicules, des mobiles, elle a rapport au mouvement plus qu'au repos : il faut savoir utiliser la force des vents, ou encore connaître la physique des fluides pour améliorer les performances du bateau; en tant que telle l'architecture navale fait lien entre l'art et la physique plus qu'elle ne met à distance la nature au moyen de l'art. L'architecture navale adapte le modèle vitruvien à la nouvelle science galiléo-cartésienne.

Comme le remarque Philippe Malgouyres (cit., p. 62), on peut être surpris par la faible place réservée dans cette bibliothèque à la peinture. La peinture, qui pourtant, à la Renaissance et jusqu'à Rubens au moins, avait joué un rôle majeur dans cette articulation art, sciences et techniques, se retire peu à peu de cette articulation, commence à s'autonomiser, à devenir non plus le paradigme de la technique mais celui des beaux-arts. 
Philippe Malgouyres note enfin l'importance des traités de jardinage (ibid., p. 59-61), l'art des jardins se développant précisément en tant que modèle d'aménagement du territoire, au point de concurrencer le modèle militaire d'aménagement par la castramétation formalisé par Vauban. L'art des jardins éclaire l'articulation arts, sciences et techniques et lui donne sa signification la plus originaire et la plus profonde. Il ne s'agit pas simplement d'être efficace, de concevoir la raison d'État dans un sens purement utilitariste. Il s'agit aussi d'embellir le monde. Dans le latin d'Alberti, embellir se dit honestare, ce qui renvoie à l'honestum du De officiis de Cicéron, de cette dignité non seulement morale mais aussi sensible de l'homme qui magnifie et transcende son utilité, le De Officiis étant sans aucun doute le livre qui exprime le mieux l'esprit des institutions à l'âge classique.

Wladimir Berelowitch, en introduction (Pierre le Grand fut-il le Pygmalion de la Russie ?, p. 20) utilise, pour caractériser le règne de Pierre le Grand, la notion de backwardness, l'avantage du retard, théorisé par l'économiste américain Alexandre Gerschenkron dans les années 1960. Plus un État part tardivement dans la course à la croissance et à la révolution industrielle, dit Gerschenkron, plus son " décollage » (take-off) est fort, et ses taux de croissance élevés. Or, la lecture de l'ouvrage ici recensé nous permet de formuler cette idée d'une façon un peu différente, dans une perspective plus politique qu'économique : en modernisant son administration plus tard que les monarchies occidentales, la Russie de Pierre le Grand nous donne à lire la formule de l'État classique de façon plus essentielle, nous aide à voir avec plus de netteté la place du savoir dans la construction de l'État classique, et nous permet de concevoir l'opérativité de la raison d'État de façon plus directe, moins symbolique, plus technique, sans son côté baroque, clérical et mystique qui, pour Pierre le Grand, affectait encore trop le règne de Louis XIV (v. sur ce point Wladimir Berelowitch, ibid., p. 17), comme si Pierre le Grand de son côté annonçait déjà, avec un demi-siècle d'avance, le despotisme éclairé de la Prusse de Frédéric II ou de l'Autriche de Marie-Thérèse, puis de Joseph II.

C'est dire enfin combien ce livre est appelé à occuper une place considérable dans l'historiographie humaniste et classique, place que renforce sa riche iconographie, le plus souvent inédite.

Pierre Caye

CNRS, Centre Jean Pépin 